\title{
TEOLOGÍA PLATÓNICA: A PROPÓSITO DE LA IDEA SUPREMA DE BIEN EN LA REPÚBLICA
}

\author{
Jean Seas Acosta \\ Universidad de Costa Rica
}

\begin{abstract}
Resumen
La idea suprema de bien constituye un problema investigativo que amerita reflexión, porque es posible determinar en Platón al primer teólogo de Occidente. Si la teología implica una reflexión racional sobre la divinidad, este es quien sienta la base de un acercamiento intelectual al dios. La teología que expresa el ateniense está matizada por la dimensión ontológica de la realidad, donde el dios puede ser comprendido como realidad-fundamento, donde es posible extraer consecuencias morales solamente a posteriori. Y esta concepción implica que su adecuada contemplación debe ser llevada a cabo por la vía de la religación, más que la religión.
\end{abstract}

Palabras clave: Platón, República, bien, Zubiri, religación

\begin{abstract}
The supreme idea of the good constitutes an investigative problem that deserves reflection because it is possible to determine in Plato, the first theologian of the west. The theology told by the Athenian is tinged by the ontological dimension of reality, where the existence of God can be understood as the foundation of all reality; therefore, it is only possible to have moral consequences to a posteriori. This conception implies that it's proper contemplation should be carried out through relegation instead of religion.
\end{abstract}

Keywords: Plato, Republic, Good, Zubiri, relegation 
La historia tiene registrado que desde las primitivas comunidades humanas ha existido un interés por establecer nexos con alguna divinidad o divinidades. De hecho, la propia invención de la escritura parece que surge del interés estrictamente religioso, pues se tiene constancia de que los "sumerios la habrían inventado con el fin de preservar su tradición religiosa" (Haartmann 2001, 73). Sin duda alguna, los elaborados discursos filosóficos que se han esgrimido en torno a la idea de dios han ocupado una gran cantidad de páginas en la historia de la filosofía y la teología, y lo seguirán haciendo, pues el dios como objeto de estudio no se muestra inmediatamente a quien investiga. La gran mayoría de la tradición teológica de Occidente mantiene un diálogo vivo con dios, mientras que la metafísica racional de corte filosófico procura mantener un diálogo de dios. La radical diferencia estriba en el "con" y en el "de" de la proposición, pues mientras que los primeros parten del supuesto de demostrar y aplicar una "realidad" trascendente a los asuntos humanos y del mundo; los segundos someten a juicio de la razón el mismo supuesto y la vía de acceso a él.

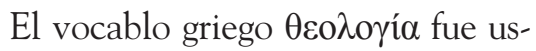
ado por primera vez en el ámbito de la filosofía por Platón de Atenas refiriéndose con él al estudio sobre el dios. Nuestra intervención es una reflexión sobre la teología natural del ateniense a partir del texto de la República (1988), para lograr establecer el vínculo y su funcionalidad dentro de la organización política de la polis griega. Dentro del corpus platónico, la dimensión teológica se puede ver como un tema transversal que ocupa al filósofo ateniense en la mayoría de sus escritos, pero tal cuestión en ninguno está tratada abierta y sistemáticamente como deseáramos. Sus referencias al ámbito de lo teológico siempre están presentadas mediante el recurso mitológico, característico en Platón cuando quiere apuntar a un conocimiento objetivo. Consecuentemente, puede comprenderse que

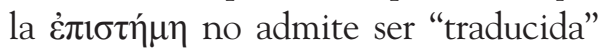
al lenguaje filosófico de la razón, porque su naturaleza es distinta de la humana, o porque no existe lenguaje que pueda dar cuenta eficaz de ella.

\section{Consideraciones en torno a la divinidad en Platón}

Antes de proponer nuestra tesis es necesario indagar sobre lo reflexionado con anterioridad, aunque hemos de admitir que pocas referencias bibliográficas existen en torno al tema. El texto de Isart (1993) quiere poner en tensión la concepción de dios que se puede extraer del pensamiento platónico respecto de la interpretación que hacen los primeros padres de la iglesia alejandrinos. La autora parte de un estudio previo que considera que en la Grecia Clásica hay dos tipos de 
corrientes religiosas: una que se puede considerar legalista e incluye toda la religión délfica con sus máximas estrictas y sus observancias inflexibles, y la segunda mística, que incluye la idea de parentesco con la divinidad. Desde su perspectiva, Platón se circunscribe en la segunda, pero la modifica un poco.

Para la autora, en Platón hay una nueva concepción de la divinidad, la manera de entenderla implica un progreso cualitativo respecto de la religión antigua y, además, un paso importante para la posterior reflexión helenística en torno a la divinidad que esgrime toda reflexión cristiana. Del corpus platónico se puede interpretar que el dios sería un trascendente, inefable, desconocido y perdurable tras la muerte del ser humano. En este sentido, habría en Platón una concepción monoteísta del dios, pero sin ser este necesariamente un dios personal.

Las consideraciones más importantes de la reflexión de Platón en torno a lo divino, para los intereses de Isart, son principalmente dos. En primer lugar está la tesis de homoiosis theós que se encuentra en el Teeteto (Platón 1988b, 176c), la cual implica una posibilidad de cierta semejanza entre el ser humano con el dios: “... la divinidad no es injusta en modo alguno; al contrario, representa el grado más alto de la justicia, de manera que ninguno de nosotros se le asemeja más que quien ha logrado llegar a ser lo más justo posible" (Platón trad. 1988b, 176c). Por ello la autora sostiene que la idea principal de Platón con su república aristócrata, es crear una élite de sabios que formaran parte del coro de los dioses, pues recuérdese que solamente el filósofo Rey podría llegar a contemplar las ideas eternas, y este es el más justo de los integrantes que componen la polis. Este aspecto preciso sería el vínculo de semejanza entre el dios y los seres humanos, lo que significa que no habría la necesidad de un Cristo que vinculara ambos planos ontológicos diametralmente opuestos: pues si se es justo, se se comparte de la esencia del dios que estaría impregnada en el alma humana, y cuya creación él mismo ha delegado a otros dioses.

La segunda tesis se circunscribe

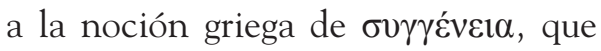
también tiene mucha relación con la anterior; pero sus implicaciones son más inmediatas y concretas. Esta idea implica la noción de lazos de parentesco que hay entre el dios y los seres humanos. Se encuentra expresamente en el Timeo (Platón 1992, 90 a-b): “... dios nos otorgó a cada uno la especie más importante en nosotros como algo divino, (...) aquello de lo que decimos que habita en la cúspide de nuestro cuerpo nos eleva hacia la familia celeste desde la tierra, como si fuera una planta no terrestre, sino celeste". Existe en Platón una necesidad y obligación por 
que el ser humano imite la divinidad en la medida de sus posibilidades; esto es, esforzarse por aproximarse a ella, pues en última instancia, el vínculo entre lo humano y lo divino dios mismo lo ha situado en el alma humana.

Por otra parte, el texto de Lagunes (2008) centra su discusión en torno a la función de la religión dentro de la polis-estado de Platón, pero no existe un esfuerzo verdadero por desarrollar suficientemente la idea. Se pretenden encontrar los fundamentos filosóficos para subrayar el papel moral y jurídico que juega la religión en el Estado, asumiendo que el ateniense introduce conscientemente la idea de un dios interior. Ese dios interior será, posteriormente, entendido como el logos de san Juan y de Justino de Roma. Lo fundamental, dice Lagunes, para entender la función de la religión dentro del pensamiento platónico viene dado por su ontología, pues su dualismo produce un ser humano habitante de dos mundos. La conclusión más importante que se desprende de su análisis implica una relación intrínseca entre filosofía y teología, y que además en Platón hay una especie de filosofía de la religión del tipo ilustrada, donde se presenta a un dios simple y trascendente.

Para lograrlo, el autor emprende un recorrido por los principales argumentos que sostiene Platón con respecto a los dioses, extraídos del texto de la República. En primer lugar se asume que la educación en la polis es sumamente importante, porque de ella resultarán productos humanos culturalizados que darán vida y funcionamiento a un Estado justo. Es por ello que para el ateniense la educación de los niños debe prescindir de fantasías contradictorias respecto a los dioses tal y como han sido tradicionalmente enseñadas por Homero y Hesíodo. Es absurdo pensar que los dioses conviven con los seres humanos, porque dioses y seres humanos son distintos por naturaleza; los seres humanos estarían relegados al plano sensible mientras que los dioses al plano inteligible. Pero tampoco se puede pensar que los dioses sean creadores de cosas malas, por ello Platón expulsa a los poetas de su polis atribuyéndoles una mala labor pedagógica, pues los dioses, siendo buenos, deben crear pocas cosas, pero buenas. La concepción platónica del dios, entonces, tendrá como atributos importantes: la bondad, la simplicidad y la veracidad.

En segundo lugar, el autor dice que hay un esfuerzo intelectual particular en Platón por comprender a la divinidad de un modo más racional. Esta sería una respuesta del ateniense a los sofistas, quienes consideraban que las divinidades eran producto de la invención humana basados en la tesis de que los dioses difieren de lugar en lugar. Aunque la religión popular griega 
es politeísta, y atribuye caracteres antropomórficos a sus dioses, se ve en el ateniense un esfuerzo por consignar los atributos entitativos de la divinidad en una unidad, librándola a su vez de todo antropomorfismo. Existe una crítica voraz en Platón respecto al antropomorfismo atribuido a los dioses populares, a tal punto que Lagunes indica que el ateniense sustituye la religión antropomórfica de los griegos, por una religión espiritual que necesariamente desemboca en la trascendencia absoluta. Esa unidad perfectamente integrada se resume en la idea suprema de bien. Hay que considerar también que en Platón el dios sería el garante de la felicidad en la polis y presenta dos características: en primer lugar dios es solo causa de lo bueno, y en segundo lugar los dioses no son encantadores que se muden así mismos, por lo cual no conducen al ser humano a extravíos. Siendo dios el garante de la felicidad en el Estado, representado en la idea suprema de bien, su veracidad no puede ser puesta en duda.

Otro aporte importante viene dado por Grube (2010). El autor realiza un estudio donde nos aproxima a una mejor comprensión de la divinidad en Platón y para ello se basa en una gran cantidad de textos del corpus platónico. Primeramente se quiere limpiar del lastre, principalmente cristiano, con el que se ha interpretado la filosofía del ateniense y proponer una lectura de la divinidad platónica con "nuevos ojos", unos ojos que tienen el horizonte filosófico que pudieron haber tenido los griegos.

Primeramente se establece que la noción griega de $\theta \varepsilon$ ćs y la idea actual de "dios" no son equivalentes y ni siquiera se corresponden. Es más, no hay palabra moderna que sirva para traducir adecuadamente el concepto de dios al que aludían los antiguos. Para los griegos $\theta \varepsilon o ́ \varsigma$ es una noción predicativa, es decir, cuando un cristiano dice que dios es amor, en primer lugar afirma la existencia de un ser misterioso, y en segundo lugar dice algo acerca de ese ser; para el griego el orden es inverso: cuando un griego afirma que el amor es dios, no está presuponiendo la existencia de ninguna divinidad misteriosa, sino diciéndonos algo acerca del amor. Por tanto, hay que comprender que cuando el griego pronuncia el vocablo $\theta \varepsilon o ́ s$, primaria y fundamentalmente, lo que quiere atribuir son los caracteres de sobrehumano, ilimitado y perenne a una cuestión intrínsecamente humana. En este mismo sentido, hay que tener presente que como hijo de su época, el ateniense también comparte la misma cosmología griega en la que se partía de una materia eterna, lo cual imposibilita pensar en un dios creador a partir de la nada, por ello, decir dios no implicaba darle realidad a la divinidad, sino elevar un concepto, como el amor o la verdad, a una categoría más elevada. 
La pregunta acerca de la naturaleza de dios, dice Grube, en Platón no admite una única respuesta, pues se trata en el fondo de dos preguntas: ¿Qué es para Platón la realidad última y absoluta? ¿Qué corresponde al aspecto dinámico de dios, al hacedor o creador de la vida? En lo que respecta a lo primero, hay que tener presente que las existencias últimas en Platón son los arquetipos; las ideas o las formas, y en este sentido son las que verdaderamente existen sin cambiar, pues son el "molde" de lo sensible. Este es un aspecto muy interesante a tomar en cuenta, porque Platón no identifica nunca a los dioses con las ideas, salvo en el Timeo 37c. Las ideas son un hecho innegable en la filosofía del ateniense. En sus diálogos ideas y dios (es) nunca se sitúan de manera contigua, por lo que hay que suponer, entonces, que los dioses empiezan a perder el primer puesto que habían mantenido originalmente, hasta el punto de considerar a los dioses subordinados por las mismas formas, pues en su contemplación encuentran ellos la felicidad. Es más, un dios es divino exclusivamente en virtud de su relación con las ideas. Es por ello que los dioses, dice Platón, aman lo justo por ser justo, y porque no podría ser de otra manera, dado que son perfectos del mismo modo que las ideas.

Es muy importante el hecho que señala Grube en cuanto a que Platón no ataca a los dioses populares, sino más bien a la concepción popular de los dioses, como ocurre en la República y las Leyes. Hay, en el Platón de los primeros diálogos, la creencia de que existen ciertas fuerzas superiores continuamente activas, pero en su etapa de madurez los dioses se acomodan a la escala eterna de valores, del mismo modo que los dioses populares obedecían a la balanza de la necesidad.

En la República los dioses parecen mantenerse aun en pie de igualdad con las ideas, pero tampoco aquí se nos otorga explicación alguna de su relación mutua. Solo aparecen dos veces en una discusión en torno al arte. En lo que respecta al Libro III de la República se critica y rechazan las narraciones de los poetas en relación con los dioses, porque ellos no los describen correctamente; parece ser que los dioses de los poetas son en realidad la encarnación de los deseos mismos del poeta. Pero también en el Libro X de la República se efectúa una crítica importante, y es en torno a la relación entre el dios y el pintor/escultor, donde Platón sitúa al pintor en una triple distancia de la verdad, por lo que su arte no puede reflejar correctamente la divinidad.

Por todo lo anterior, hay que considerar el carácter mítico de los dioses, pues ellos no son mencionados en discusiones acerca de la teoría de las formas, el aspecto más importante 
del pensamiento de Platón. Las ideas y los dioses representan dos principios diferentes del mundo suprasensible, la realidad última y la causa primera del movimiento, principios que Platón mantuvo separados al afirmar continuamente la prioridad del primero. Hay un grave error al considerar que las ideas en Platón funcionan como dios, tampoco las ideas son los pensamientos de dios. Pues, en primer lugar, las ideas preexisten al dios, quien crea a partir de ellas y, en segundo lugar, se debe comprender que el dios piensa las ideas porque existen, y no existen porque él las piense. Consecuentemente, la idea suprema de belleza, verdad y bien no debe corresponderse nunca con el dios.

El texto de Grondin (2010) quiere centrar la atención en la idea de la funcionalidad de la religión dentro del pensamiento platónico. Su tesis principal es que el ateniense prefiguró una religión que es ante todo moral. Para el autor lo importante en su reflexión es encontrar el vínculo existente entre toda religión y la felicidad humana, porque una y otra irán a menudo a la par por un largo trecho de la filosofía de la religión. No obstante, nos parece que el autor no indaga necesariamente la concepción del dios en Platón, sino solo su efecto cultural, es decir, la religión.

Platón encuentra, dice Grondin, las pruebas de la existencia de la divinidad en el orden del mundo como todos los griegos clásicos y los autores latinos, basado en el movimiento regular de los astros. La evidencia más patente de la existencia del dios reside en la constancia admirable de los astros, cuyo movimiento eterno es tan perfecto que no puede tener otra causa que los dioses. Pero no solamente desde el punto de vista cosmológico se entiende la divinidad, sino también en sus reflexiones de orden ontológico. La prueba consiste en la primacía del alma sobre el cuerpo: si el cuerpo es movido por el alma es porque es segundo respecto a ella, y las almas creadas por el dios han existido antes de sumergirse en tal o cual cuerpo. Al formular estas pruebas, el ateniense es el primero en esgrimir una teología racional, o filosofía de la religión entendida como justificación argumentada de las grandes creencias religiosas.

En sus reflexiones, Platón no ataca únicamente a quienes niegan la existencia de los dioses, critica también a quienes piensan que los dioses no se ocupan de los seres humanos, pues para él esto significa acusarlos de holgazanería y negligencia, lo cual es absurdo porque son perfectos. Consecuentemente, el mejor culto que se puede ofrecer a los dioses, según Platón, es practicar la justicia, puesto que los dioses son justos; contrario a lo que cree la religión popular que pretende agradar a los dioses con cultos, 
sacrificios y plegarias, dado que la religión popular griega ve en los dioses seres maleables. No hay ninguna duda, a los ojos de Platón, de que este es el culto que Sócrates practicó mejor que cualquier otro griego, al punto que en el libro de las Leyes se asume que deberán dictarse estrictas leyes contra la impiedad de los hombres, porque si el hombre cree que los dioses existen, no cometerá nunca un acto impío.

En lo que respecta al texto de González (2008), se parte de que en Platón existe una teología natural, sin embargo, no desarrolla suficientemente la nación porque no es el interés que persigue en su obra. La teología natural o teodicea es la parte de la metafísica que estudia a dios. En Platón hay una teodicea, porque sus reflexiones en torno al dios vienen dadas por la misma razón que critica las formas tradicionales de la religión popular. Históricamente, todos los filósofos han afrontado el problema de dios, de un modo o de otro. No ha existido ni un solo filósofo, apunta el autor, que no haya escrito sobre dios, incluso los que con sus principios filosóficos pretenden no dejar lugar a dios, desplazándolo, negándolo y hasta borrando su mismo nombre. Por lo anterior, se puede decir que pensar en torno al dios es una característica común a todas las doctrinas metafísicas por muy divergentes que puedan ser, casi todas están de acuerdo en la necesidad de hallar la causa primera de lo que es. En lo que respecta al pensamiento de Platón, el dios sería el fundamento de la realidad, al que se le predica el atributo de la bondad y la sabiduría.

\section{Considerando el dios de Platón}

En los primeros diálogos el ateniense mantiene una posición teológica que bien podemos llamar convencional, pues admite que hay dioses sobrenaturales e inmortales. No obstante, cuando desarrolla su República se nota un cambio de concepción en torno a su idea de dios. Hemos de limitarnos al enfoque hermenéutico desde la perspectiva de análisis de una teodicea, para comprender cómo es el dios que dibuja Platón. Por lo que a fin de ser fiel a su pensamiento teológico, debemos aproximarnos al texto dejando de lado todo el lastre ideológico del cristianismo. Este último ha considerado que la idea de bien, que se desarrolla en el libro VII de la República, se corresponde con el dios del cristianismo, y tales ideas deben situarse en el pensamiento de Clemente de Alejandría y Justino. Sin embargo, hay dos contradicciones que surgen inmediatamente, una es de orden cronológico y la otra de orden lógico.

En cuanto a la primera tenemos que admitir que es humanamente imposible que el griego elabore su teología en vistas de fundamentar filosóficamente el cristianismo, puesto que el 
cristianismo históricamente ha constituido una reflexión intelectual que se ha hecho en el tiempo respondiendo a problemas concretos que se le aparecen, a fin de defender los dogmas en los que cree. El cristianismo surge posterior a Platón, por lo que el ateniense no tiene necesidad de estar consciente de ello en sus reflexiones. No obstante, bien puede pensarse que los padres de la Iglesia usen las reflexiones teológicas del ateniense para fundamentar racionalmente al cristianismo, en un acto ulterior, pero esto lleva consigo un problema mayor al que señalamos anteriormente, y es el de orden lógico. Platón nunca asume explícitamente en la República que la idea de bien sea intercambiable por su noción de dios, pero el Sol sí parece poseer esa característica. Sin embargo, dice algo muy importante en torno a ella: "a las cosas cognoscibles les viene del Bien no solo el ser conocidas, sino también de él les llega la esencia, aunque el Bien no sea esencia, sino algo que se eleva más allá de la esencia en cuanto a dignidad y a potencia” (Platón 1988a, 509c). Consecuentemente, el dios del cristianismo no puede corresponderse a la idea de bien porque dicha idea carece de esencia misma, y desde la perspectiva dogmática del cristianismo la esencia de dios implica su existencia.

El dios en Platón constituye, verdaderamente, un problema investigativo que amerita reflexión. Primero, porque tenemos noticia de él por vía mitológica, y mediante esta vía se condensan las ideas más importantes de la filosofía del ateniense. Y en segundo lugar, porque los apologetas cristianos centraron su fundamentación teológica, asumiendo que la idea de bien es intercambiable con el dios en el que creen. Ese dios del cristianismo parece asemejarse más en sus funciones al $\Delta \eta \mu$ iovprós del Timeo, pero dicho personaje en Platón no es considerado nunca como el dios. Aunque el Timeo sea un verdadero discurso sobre el $\Delta \eta \mu 10 v \rho \gamma o ́ \varsigma$, no es una aproximación teológica que verdaderamente nos interese, porque el $\Delta \eta \mu 10 v \rho \gamma o ́ s$ ordena la materia eterna que se encuentra en caos, conduciéndola del desorden al orden, y le imprime la forma que existe eternamente en las ideas del mundo inteligible; desde esta perspectiva el Timeo no da cuenta de la jerarquía de las ideas (Fouillée 1943), por lo que la República contiene más elementos para aproximarnos a establecer una ontología del dios de Platón.

Es posible extraer de la República la tesis de que el dios no es un ente exterior al Universo, pero sí se encuentra en un lugar específico y, por ello, no necesariamente debemos situarnos en un inmanentismo. Es más, la crítica de Platón a los poetas en torno a que dibujan un dios a conveniencia de ellos puede ser fundamental para aproximarnos a interpretar que el dios 
del ateniense solo adquiere realidad en tanto que alguien lo piense, pero se debe pensar de una manera correcta. Por ello, es conveniente afirmar que dicho dios es un ente necesario de razón que tiene una función específica en la república que organiza Platón. Hablando sobre los deberes de los guardianes, y las posibles desviaciones del bien que puedan surgir en ellos cuando ejerzan la administración de la polis, Platón dice: "les diremos que, gracias a los dioses, cuentan siempre en el alma con oro y plata divina y que para nada necesitan de la humana, y que sería sacrílego manchar la posesión de aquel oro divino con la del oro mortal (Platón 1988, 416e). Ese "les diremos" es lo más importante de la reflexión teológica. El ateniense realiza una verdadera teología fundamentada en la razón, en cuanto admite que del dios provienen las virtudes necesarias para organizar un Estado justo. Y el mismo Sócrates incluso puede decir "que algún dios ha concedido a los seres humanos estas dos artes, la de la música y la de la gimnasia, con miras a estas dos cosas: la fogosidad y el ansia de saber" (República 411e). Por tanto, el dios es la justificación que legitima la racionalidad en el momento de administrar el Estado justo.

En su República Platón quiere que cada ciudadano ejerza la función que le es propia, pues eso es lo justo, pero para ello debe tener un administrador que dirija la polis con miras al bien. Después de un proceso educativo específico, en donde para llegar a ser filósofo rey debe pasarse por una educación basada en matemáticas, geometría, astronomía, y dialéctica, este será el mejor ciudadano capacitado para dirigir los asuntos del Estado. Pero el filósofo rey parece que no tiene que compartir la misma noción de dios que el resto de los ciudadanos de la polis, pues hablando del hombre justo, aquel que ha llegado a contemplar la idea suprema de bien, Platón dice que "también los adulterios y la negligencia respecto de los padres y del culto a los dioses convendrían a cualquier otro menos al hombre de que hablamos" (Platón 1988, 443a). Consecuentemente, tenemos que el culto al dios en la República actúa en dos planos distintos y ejerce funciones distintas: el filósofo rey, quien ha contemplado la idea suprema de bien, es quien "crea" el culto y la idea de dios que tiene que comunicar a los ciudadanos con miras al bien. Los ciudadanos tendrán que admitir por verdadero a ese dios, pues quien lo ha comunicado ha sido el filósofo, quien ha contemplado al bien. Por lo tanto, el dios que esgrime Platón es un ente de razón creado para fundamentar las decisiones del administrador de la polis, y aquel que legitima el rumbo de la administración.

Ahora bien, que el filósofo rey contemple la idea de bien conlleva 
grandes responsabilidades, pues no puede usar la idea del dios para legitimar cualquier posible desequilibrio entre sus parte del alma. Hemos afirmado que el dios del cual nos habla Platón no puede entenderse nunca como las ideas, pero las ideas son divinas en cuanto posee o son de una categoría más elevada que la humana. "Entonces, en cuanto el filósofo convive con lo que es divino y ordenado se vuelve él mismo ordenado y divino, en la medida que esto es posible al hombre" (Platón 1988a, 500 c-d). Es clara la referencia con un dios que se admite en el Estado por necesidad de legitimar el orden de la polis, pero un dios esgrimido únicamente por el pensamiento desvirtúa la naturaleza misma de la idea suprema de bien, cuando hemos apuntado que entre ambas existe un vínculo necesario.

Asumir que en la teología de Platón el dios solo es un ente de razón que legitima la administración de la polis, es no darle el verdadero valor a la divinidad. Ese dios que es construido mentalmente por el filósofo rey no solo debe tener implicaciones en el ámbito ético con miras a una adecuada administración, sino también en el ámbito ontológico. La alegoría de la caverna contiene algunas pistas importantes para su adecuada interpretación. Pregunta Sócrates: “¿A cuál de los dioses que hay en el cielo atribuyes la autoría de aquello por lo cual la luz hace que la vista vea?, responde Glaucón: Al mismo que tú y que cualquiera que los demás, ya que es evidente que preguntas por el sol" (Platón 1988a, 508a). Esta directa analogía del dios más importante con el sol es crucial en nuestro análisis. Pues del mismo modo que el sol ilumina la realidad y hace que aparezca, el dios de Platón debe ser el fundamento de la realidad. La luminosidad del sol sobre la materia sensible, primera vía de acceso al conocimiento verdadero que tiene su culminación por vía intelectual en la idea suprema de bien, solamente hace visible lo que estaba antes oculto al esclavo de la caverna. Por ello, asumir que el dios y la idea suprema de bien sean correspondientes, no es sino ver en la obra

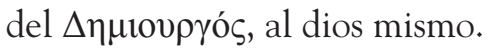

\section{Dios como fundamento de la realidad}

En Zubiri (1994) encontramos el mismo problema al que se enfrenta Platón respecto a sus reflexiones teológicas. La consideración de dios como fundamento de la realidad también incluye la dimensión ética del ser humano. Hay que establecer, en primer lugar, que a dios solamente llegamos por la vía de la religación, que no es una mera vinculación ni es un sentimiento de dependencia, sino la versión constitutiva y formal al poder de lo real. La vía de la religación no es formalmente ni cósmica ni antropológica, pero es ambas cosas por eminencia, y es la única por la cual 
llegamos a dios en cuanto dios. Tanto en Zubiri como en Platón existe esta condición de acceso al dios, que alude a una necesidad epistemológica por encontrar el fundamento de lo real. Pero religación no debe confundirse nunca con el término religión, pues esta última implica la necesidad de volver a unir lo que antes estuvo unido. Ni en Zubiri ni en Platón el dios puede separarse de lo real en tanto que real, porque el dios es constitutiva y formalmente lo real. Ello resultará que en ambos pensadores no exista una necesaria manifestación cultural de dios, una religión, puesto que el dios no debe ser comprendido como una especie de entidad humana que prescinde de toda imperfección. De aquí, la enérgica eliminación de dos errores graves entre los cuales se ha solido oscilar fácilmente. El primero es el error de pensar que la presencia formal del dios en las cosas hace, de estas, momentos de la única realidad del dios, lo que ha llevado a admitir la tesis panteísta; esto es imposible, el dios está formalmente en las cosas, pero "haciendo" que ellas sean en dios realidades distintas de él. Y el segundo error es de signo opuesto al primero. Consiste en pensar que, por ser radicalmente distinto de las cosas, el dios es el gran ausente, el gran extranjero del mundo. Está, en todas ellas, la forma extrema de concebir al dios como "más allá" de las cosas (Zubiri 1994, 176).
Si hemos de considerar que el dios es fundante de la realidad, debemos asumir que necesariamente tiene que ser una realidad suprema; pero no un ente supremo, y esta consideración implica, lógicamente, que el dios no puede ser una cosa más en el mundo ni allende a él. El dios necesariamente debe estar más allá del ser, porque la entificación de lo real, dice Zubiri, es un acto ulterior a lo real, puesto que las cosas necesitan primero ser reales para poder ser, ulteriormente, entes. Zubiri observa que todas las cosas son reales, pero ninguna es "la" realidad. Pero "la" realidad es real porque determina físicamente haciéndose ser relativamente absoluta. Luego, existe necesariamente otra realidad en que se funda "la" realidad, y esta realidad no es una cosa concreta más, porque no es "una" realidad, sino el fundamento de "la" realidad (Zubiri 1994, 148). De ahí se desprende que dios sea la realidad última, sin él las cosas no serían reales. Esta idea compete mucho con la ontología platónica y la preeminencia del mundo inteligible sobre el sensible.

Por eso podemos afirmar que la idea de bien puede ser considerada como el dios platónico, en tanto que en dicha idea se reúnen todas las ideas del mundo inteligible, pero debemos aclarar que ese dios no posee esencia, pero de él emana la esencia de las ideas. Desde este punto de vista, el bien otorga realidad a lo real, las ideas, que 


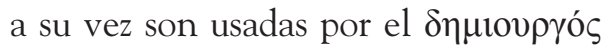
para concretar lo sensible. Consecuentemente, tanto para el ateniense como para el vasco, dios, como fundamento de lo real, no puede ser tampoco más que uno y único.

Desde la perspectiva de Zubiri, dios está presente en las cosas reales como formalidad fundante intrínseca, y ello no significa que entre dios y las cosas no haya distinción real, sino significa, tan solo, que no hay "separación” ni física ni metafísica. Este carácter según el cual dios está presente en las cosas con una presencia intrínseca y formal, y que, sin embargo, las cosas no son dios, es lo que llama el vasco trascendencia de dios "en" la realidad" (Zubiri 1994, 174). Pero esta trascendencia no quiere decir "más allá" de las cosas, no es un allende las cosas. Dios está formal e intrínsecamente en ellas dándoles de suyo lo único que posee, es decir, está dándoles la realidad. Que dios esté en las cosas, pero que no exista identidad de las cosas con dios, es justo la noción de trascendencia que quiere señalar Zubiri. En Platón parece funcionar del mismo modo, el dios está presente en las cosas como una especie de esencia degradada que se imprime en lo sensible desde el mundo inteligible. Dicha reunión de todas las ideas en la idea suprema de bien es justamente lo que la convierte en la realidad absoluta, pues en la ontología del ateniense, la idea suprema de bien no es una idea más del mundo inteligible, sino el principio de realidad que reúne todas las ideas. En la teología platónica, al dios se llega por medio del intelecto que asciende desde el mundo sensible hasta el inteligible.

Esta presencia del dios en las cosas, pero distinto de ellas, coloca en un absurdo la tesis inmanentista y la trascendentalista como ordinariamente las ha solido entender la filosofía. Hemos visto que, en ausencia del dios, la realidad no sería real, o lo que es lo mismo, sin el dios nada sería. Pero descubrir al dios no significa demostrar su existencia. Recíprocamente, demostrar la existencia del dios no significa que el razonamiento sea "el" camino para descubrir al dios. La demostración de la existencia de dios no es primariamente una exigencia lógica que se esgrime frente a quienes no creen en el dios. Desde el punto de vista semántico, el descubrimiento alude a la noción de develación, y develar es hacer visible lo existente. Como hay que mostrar lo existente, porque su contrario nos hace caer en absurdos, tanto Platón como Zubiri nos muestran la realidad, las cosas, y apuntan a que en ellas hay algo que debe hacerlas reales, pues aunque Platón no lo admita de primera entrada, el conocimiento humano primero indaga lo sensible y solo ulteriormente lo inteligible. Como las cosas son reales, pero no son la realidad, ese algo debe ser el dios como fundamento de lo real. 
Pero en el pensamiento platónico existe una particularidad, puesto que a la idea suprema de bien solo puede tener acceso el filósofo rey, este es quien debe "traducir" a los ciudadanos una teología que responda a los intereses administrativos de la polis, con miras al bien común. Por ello, en la polis el dios debe ser un ente de razón, que se corresponda a la idea de bien. Es el filósofo rey, porque ha contemplado el bien, quien sabe que el dios es el fundamento de la realidad, primero inteligible $y$, posteriormente, sensible por suerte de degradación.

Zubiri, al afirmar la trascendencia mundanal, y Platón, al afirmar que la idea suprema de bien le otorga esencia a las ideas del mundo inteligible, nos sitúan en un realismo radical, aun cuando los supuestos sean metafísicos propios del intelecto. Justamente la robustez metafísica yace en el hecho de no aludir al dios como una cosa más en el mundo, y más aún, un allende al ser, pero que se encuentra constitutiva y formalmente en todas las cosas otorgándoles lo que tiene de suyo, realidad.

Ciertamente la idea suprema de bien puede corresponderse al dios platónico, pero este dios no puede corresponderse nunca al dios de las religiones monoteístas con mayor cantidad de seguidores. Lejos está el ateniense de querer esgrimir alguna religión, puesto que dios solamente es la suprema realidad que le otorga realidad a lo demás. Sin embargo, es factible pensar que el filósofo rey en la República deba propiciar algún tipo de culto en virtud del dios, a fin de legitimar su administración, en vista de que solamente él puede tener el conocimiento de la función del dios. Tenemos, por tanto, que el dios platónico actúa en dos realidades, la realidad real, la del filósofo rey que contempla la idea de bien, y la realidad aparente, la de los ciudadanos que necesitan una divinidad que legitime el poder y las decisiones del filósofo. Es por ello que dice Platón que es "el filósofo el único capaz de soportar la contemplación de lo que es, y lo más luminoso de lo que es, que es lo que llamamos Bien" (Platón 1988a, 518c-d). Consecuentemente el mito de Er consignado en el Libro X de la República daría luces necesarias de la función del dios en el Estado. Dios es quien legitima las decisiones administrativas en la polis, el gran vigilante de los ciudadanos en virtud de la concordia en la polis. Pero, para el filósofo rey, la idea suprema de bien es el fundamento de la realidad, que no admite religión, porque entre las cosas y dios no hay separación, sino más bien religación. 


\section{Bibliografía}

Fouillée, Alfred. 1943. La filosofía de Platón. Buenos Aires: Ediciones Mayo.

González, Ángel. 2008. Teología natural. Navarra: EUNSA.

Grondin, Jean. 2010. La filosofía de la religión. Barcelona: Herder.

Grube, George. 2010. El pensamiento de Platón. Madrid: Gredos.

Haartmann, Harald. 2001. Historia universal de la escritura. Madrid: Gredos.

Isart, María. 1993. Evolución de la relación hombre/dios desde Platón al siglo II. Anuario de Estudios Filológicos, 16, 207-14.
Lagunes, Óscar. Mayo-agosto, 2008. La religión en Platón. Un acercamiento a su filosofía de la religión. Revista Philochristus, 6, 18-23.

Platón. 1988a. Diálogos IV: La República. Madrid: Gredos.

Platón. 1988b. Diálogos V: Teeteto. Madrid: Gredos.

Platón. 1992. Diálogos VI: Timeo. Madrid: Gredos.

Zubiri, Xavier. 1994. El hombre y Dios. Madrid: Alianza Editorial. 
